HRT- 3 Morfi el ds reference pl ane: effect on rimarea repeat ability and identification of progr essi on

\begin{tabular}{|l|l|}
\hline 著者 & $\begin{array}{l}\text { Asaoka Ryo, St r out hi di S N G, Kappou V, } \\
\text { Gar di ner S K, Gar way- Heat h D F }\end{array}$ \\
\hline $\begin{array}{l}\text { j our nal or } \\
\text { publ i cat i on t i t l e }\end{array}$ & Briti sh Jour nal of Opht hal nol ogy \\
\hline vol une & 93 \\
\hline number & 11 \\
\hline page r ange & $1510-1513$ \\
\hline year & $2009-06-16$ \\
\hline URL & ht t p: //hdl . handl e. net /10271/2417 \\
\hline
\end{tabular}




\title{
HRT-3 Moorfields reference plane: effect on rim area repeatability and identification of progression
}

\author{
R Asaoka, ${ }^{1,2}$ N G Strouthidis, ${ }^{1,3}$ V Kappou, ${ }^{1}$ S K Gardiner, ${ }^{3,4}$ D F Garway-Heath ${ }^{1}$
}

\begin{abstract}
${ }^{1}$ NIHR Biomedical Research Centre for Ophthalmology, Moorfields Eye Hospital NHS Foundation Trust and UCL Institute of Ophthalmology, London, UK; ${ }^{2}$ Department of Ophthalmology, Hamamatsu University, School of Medicine, Shizuoka, Japan; ${ }^{3}$ Optic Nerve Head Research Laboratory, Portland, Oregon, USA;

${ }^{4}$ Discoveries in Sight, Devers Eye Institute, Portland, Oregon, USA

Correspondence to: Dr D F Garway-Heath, Glaucoma Research Unit, Moorfields Eye Hospital, 162 City Road, London EC1V 2PD, UK; david.garwayheath@moorfields.nhs.uk
\end{abstract}

Accepted 7 May 2009 Published Online First 16 June 2009

\author{
ABSTRACT \\ Aims: To assess the effect of the Moorfields Reference \\ Plane on Heidelberg Retina Tomograph (HRT) rim area \\ repeatability and its effect on progression rates using an \\ event analysis.
}

Methods: The HRT reference plane (RP) defines structures above as "rim" and below as "cup." The Moorfields RP applies the Standard RP (located $50 \mu \mathrm{m}$ posterior to the temporal disc margin) at baseline and maintains the distance between the Standard RP and the reference ring (located in the image periphery) for followup images. The Moorfields RP was applied to an HRT test-retest dataset, and rim area repeatability coefficients were calculated. Repeatability coefficients were compared between the Moorfields, Standard and 320 llocated $320 \mu \mathrm{m}$ posterior to the reference ring) RPs. The Moorfields RP was applied to HRT images from 198 ocular hypertensives, acquired over 6 years. HRT progression required rim area baseline/follow-up differences exceeding the repeatability coefficient in two or more sectors, with confirmation in at least one of two consecutive images. Field progression was assessed using Advanced Glaucoma Intervention Study criteria.

Results: The Moorfields RP improved rim area repeatability compared with the Standard RP; repeatability was similar between the Moorfields and the 320 RP. The frequency of identified progression using Moorfields RP was $40 \%$ compared with $28 \%$ for the 320 RP. There was a greater percentage with concurrent field progression -15.1\% (Moorfields RP) compared with 12.1\% (320 RP). Conclusions: Although rim area repeatability was similar using the $320 \mathrm{RP}$ and the Moorfields RP, the latter resulted in greater rates of detection of change.

Semiautomated optic nerve head $(\mathrm{ONH})$ and retinal nerve fibre layer (RNFL) imaging devices have been commercially available for over a decade. These devices have potential applications in the management of patients with, or at risk of, glaucoma, both in assisting the clinician in the discrimination of normal optic discs from glaucomatous optic discs, ${ }^{1-4}$ and in the monitoring of progression..$^{5-10}$ No consensus, however, yet exists as to how best to integrate these new technologies into clinical practice and what are the optimal operating criteria to enable their full potential to be realised.

The Heidelberg Retina Tomograph (HRT, Heidelberg Engineering, Heidelberg, Germany), which works on the principle of confocal scanning laser ophthalmoscopy, is the longest established of the scanning imaging devices used in glaucoma management. All new HRT software developments (the Explorer operational software is now in its third version) enable backward compatibility, so that images acquired using older versions of the device, such as the original HRT (now referred to as HRT "Classic"), can be analysed.

One approach to monitoring progression using the HRT is to monitor change in a particular stereometric parameter (such as rim area; RA) over time. A number of progression algorithms have been described which assess RA change. ${ }^{56}$ RA has been proposed as a good marker for progression, as it has been shown to be a repeatable, reliable and clinically meaningful HRT parameter. ${ }^{11-13}$

The greatest challenge in detecting progression is the ability to discriminate true change (disease process) from measurement variability. RA variability is strongly influenced by the position of the reference plane (RP) ${ }^{11}{ }^{13} 14$ The RP is parallel to, and below, the retinal surface, and is used to define structures above the plane as "rim" and below as "cup." The default RP in the Heidelberg Explorer software is the standard RP (SRP), located $50 \mu \mathrm{m}$ posterior to the temporal disc margin. An alternative $\mathrm{RP}$, the $320 \mathrm{RP}$ is located $320 \mu \mathrm{m}$ posterior to the reference ring (which is located in the image periphery) and has been shown to result in less RA variability. ${ }^{12}$ On this basis, our group has previously proposed the use of the $320 \mathrm{RP}$ with RAbased progression algorithms. ${ }^{69}$

We have recently described and tested a novel RP, the Moorfields RP (MRP). ${ }^{15}$ The MRP applies the Standard RP in the baseline image and then keeps the height difference between the baseline $\mathrm{RP}$ and the reference ring constant for follow-up images (fig 1).

The aim of this study was to further investigate the performance of the MRP. First, the effect of the MRP on RA repeatability was assessed. Second, its effect on the detection of glaucoma progression was investigated by applying the MRP to an HRT RA event analysis (EA) algorithm.

\section{MATERIALS AND METHODS}

\section{Assessment of rim area repeatability}

Repeatability was assessed by applying the MRP to HRT images acquired as part of an HRT test-retest study which has been described in detail elsewhere. ${ }^{11} 12$ In summary, 43 eyes with ocular hypertension (OHT) and 31 with primary openangle glaucoma (POAG) were imaged by two experienced observers using the HRT Classic on the same date, and on a second date within 6 weeks of the date of the first image acquisition. Subjects had no previous history of intraocular surgery and had prior experience of $\mathrm{ONH}$ imaging using the HRT. The acquired HRT Classic single topographies were imported as HRTport files into a beta version of HRT Explorer Version 3.1.2.0, 


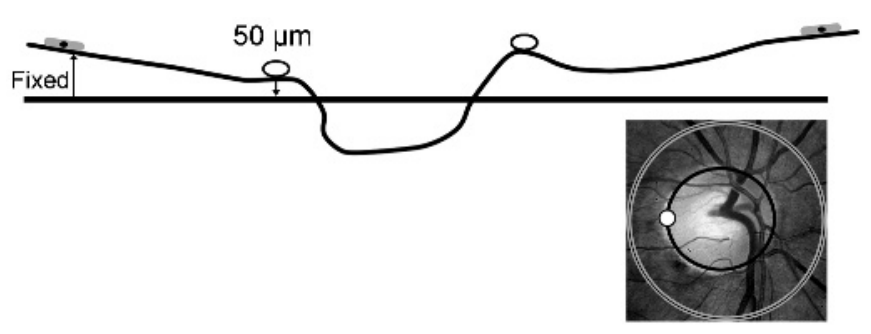

Figure 1 Location of the Moorfields reference plane. The height of the reference plane in the baseline image is the same as for the standard reference plane, located $50 \mu \mathrm{m}$ posterior to the temporal disc margin (white disc with black outline). The height difference between the baseline reference plane and the reference ring (black circle with grey outline) is kept constant for follow-up images. Figure previously published in Strouthidis NG, Gardiner SK, Sinapis C, et al. The spatial pattern of rim loss in ocular hypertension. Invest Ophthalmol Vis Sci 2009;50:3737-42. (C) Association for Research in Vision and Ophthalmology.

incorporating the MRP, with which mean topography images were generated.

The British Standards Institution definition of measurement error, the repeatability coefficient (RC), was used to quantify RA repeatability. ${ }^{16}$ Ninety-five per cent of intertest differences are expected to lie within the value of the RC. RC was calculated as:

$$
\mathrm{RC}=2^{*}\left(\sqrt{\frac{\sum \text { (observation 1-observation2) }^{2}}{\mathrm{n}(\text { observations) }}}\right)
$$

Interobserver/intervisit RA RCs (using the MRP) were calculated for the six predefined HRT sectors, namely superotemporal (ST), inferotemporal (IT), temporal ( $\mathrm{T})$, superonasal $(\mathrm{SN})$, inferonasal (IN) and nasal (N). HRT image quality was defined according to the mean pixel height standard deviation (MPHSD). Each subject eye was ranked by MPHSD (taken as the mean MPHSD of observation 1 and observation 2) with good-quality images ranked in the upper tertile, medium-quality images in the middle tertile and poor-quality images in the lower tertile. Sector RA RCs generated using the MRP were compared against previously published RCs generated using the SRP and 320 RP. ${ }^{12}$

\section{Assessment of HRT progression}

The interobserver/intervisit RA RCs generated using the MRP were used as the basis for "criteria for change" in an HRT event analysis progression algorithm. ${ }^{6}$ In the current study, HRT progression was flagged if a decrease in sector RA (baselinefollow-up) exceeded the sector RA RC for that level of image quality (taken as the mean of the baseline and follow-up MPHSD) in two or more sectors and confirmed in at least two out of three consecutive images. Significant improvement was defined using the same criteria, except requiring an increase in sector RA from baseline to follow-up. This strategy was classified as event analysis 2 (EA2) in our previous report. ${ }^{6}$

The EA2 strategy was applied to HRT mean topographies acquired using HRT "Classic," from 198 OHT subjects and 21 control subjects followed prospectively with regular HRT and VF testing (1994 to 2001), originally recruited to a betaxolol versus placebo study. ${ }^{17}$ In brief, OHT was defined as an IOP $>22 \mathrm{~mm} \mathrm{Hg}$ and $<35 \mathrm{~mm} \mathrm{Hg}$ on two or more occasions within a 2-week period and a baseline mean Advanced Glaucoma Intervention Study (AGIS) VF score of 0 (Humphrey Field Analyzer, full-threshold 24-2 program).${ }^{18}$ Control subjects were recruited from senior citizens groups or were the spouses or friends of subjects in the OHT cohort; they were not attending the eye clinic as patients and were not seeking care or undergoing check-ups. ${ }^{8}$ Controls had a baseline IOP of less than $21 \mathrm{~mm} \mathrm{Hg}$ and normal baseline VF test results (same criteria as in the OHT group), and were excluded if there was a self-reported family history of glaucoma or any coexistent ocular or neurological pathology. There was no significant age difference between the OHT and control groups $(p=0.09$, Student $t$ test), and both groups had similar ethnic backgrounds, both being predominantly (>95\%) of European descent. In the current study, the same eye was selected for analysis as had been randomised in the original study. The OHT eye randomisation was based on risk to glaucomatous conversion, classified according to pattern electroretinogram results, IOP and cup-to-disc area ratio at the time of recruitment; ${ }^{17}$ control eyes were selected by simple randomisation.

HRT mean topographies were generated and analysed using a beta version of Heidelberg Explorer Version 3.1.2.0 (Heidelberg Engineering, Heidelberg, Germany). The MRP was used for all analyses. A single observer (NGS) drew contour lines onto the baseline mean topographies. Contour lines were exported automatically to follow-up images. Manual alignment was used to correct for automatically "misplaced" contour lines or if there was a magnification change. Each subject had a minimum of five HRT mean topographies, with all image qualities included except where satisfactory contour line alignment could not be achieved. In total, eight mean topographies were excluded from the study, either as a result of "double imaging" or if the image was so grainy as to prevent adequate visualisation of Elschnig's ring. The baseline HRT image was the first mean topography available for each subject, and the baseline VF was taken as the VF test coinciding with, or nearest in acquisition date to, the baseline HRT mean topography.

In the absence of an accepted gold standard for defining glaucomatous progression, two proxy measures were used to generate an estimated "specificity" for EA2 using the MRP. The first proxy measure was the number of control subjects (of 21) progressing, and the second was the number of subjects (of 219) demonstrating significant improvement using the applied "change" criteria. The number of OHT subjects identified as progressing ("positive hit rate") was compared with the previously published results for the $320 \mathrm{RP}$ applied to the same OHT cohort. ${ }^{6}$ HRT progression was compared with VF progression, which was identified if the AGIS VF score increased from 0 to 1 or more and was reproducible in three consecutive VF tests in the same region of the VF. ${ }^{18}$ Previous studies have estimated the specificity of the AGIS VF strategy at $91-100 \% .{ }^{19-21}$

The study adhered to the tenets of the Declaration of Helsinki and had local ethical committee approval, in addition to subjects' informed consent. All statistical analyses were performed using Medcalc Version 7.4.2.0 (Medcalc Software, Mariakerke, Belgium).

\section{RESULTS}

Interobserver/intervisit RA RCs for the SRP, 320 RP and MRP are shown in table 1.

Regardless of image quality, RA repeatability was generally poorer (higher RC values) for the SRP than with the $320 \mathrm{RP}$ and MRP. Differences were equivocal between RA repeatability using the $320 \mathrm{RP}$ and the MRP, except in the temporal sector of 
Table 1 Interobserver/intervisit rim area repeatability coefficients $\left(\mathrm{mm}^{2}\right)$ for the standard reference plane, 320 reference plane and Moorfields reference plane

\begin{tabular}{lllllll}
\hline & T & N & ST & IT & SN & IN \\
\hline Good image quality (MPHSD <21) & & & & & & \\
$\quad$ Standard reference plane & 0.043 & 0.033 & 0.011 & 0.018 & 0.017 & 0.007 \\
$\quad 320$ reference plane & 0.050 & 0.014 & 0.012 & 0.026 & 0.008 & 0.007 \\
$\quad$ Moorfields reference plane & 0.031 & 0.023 & 0.013 & 0.012 & 0.009 & 0.012 \\
Medium image quality (MPHSD 21-35) & & & & & & \\
$\quad$ Standard reference plane & 0.083 & 0.047 & 0.036 & 0.047 & 0.018 & 0.032 \\
$\quad 320$ reference plane & 0.058 & 0.031 & 0.026 & 0.026 & 0.022 & 0.015 \\
$\quad$ Moorfields reference plane & 0.082 & 0.024 & 0.031 & 0.025 & 0.016 & 0.015 \\
Poor image quality (MPHSD >35) & & & & & & \\
$\quad$ Standard reference plane & 0.134 & 0.092 & 0.066 & 0.065 & 0.052 & 0.032 \\
$\quad 320$ reference plane & 0.109 & 0.068 & 0.057 & 0.046 & 0.049 & 0.029 \\
$\quad$ Moorfields reference plane & 0.161 & 0.071 & 0.055 & 0.049 & 0.042 & 0.024 \\
\hline
\end{tabular}

IN, inferonasal Heidelberg Retina Tomograph sector; IT, inferotemporal Heidelberg Retina Tomograph sector; MPHSD, mean pixel height standard deviation; N, nasal Heidelberg Retina Tomograph sector; SN, superonasal Heidelberg Retina Tomograph sector; ST, superotemporal Heidelberg Retina Tomograph sector; T, temporal Heidelberg Retina Tomograph sector.

medium- and poor-quality images where the MRP generated RCs of considerably greater magnitude than the $320 \mathrm{RP}$. A formal comparison of the intertest RA differences used to generate the RCs was performed using the generalised estimating equation technique. ${ }^{22}$ This method is related to the standard least-squares linear regression but adjusts for the fact that the RA intertest differences from the six sectors are correlated, as they are derived from the same subject (in each of the 74 data sets). Using the MRP, the mean intertest RA difference was $0.001 \mathrm{~mm}^{2}$ greater than with the $320 \mathrm{RP}$, although this did not reach statistical significance $(p=0.229)$. Using the SRP, the mean intertest RA difference was $0.004 \mathrm{~mm}^{2}$ greater than with the $320 \mathrm{RP}(\mathrm{p}=0.04)$ and $0.003 \mathrm{~mm}^{2}$ greater than with the MRP $(p=0.037)$.

The baseline demographics and characteristics of subjects included in the progression analysis are summarised in table 2.

The specificity for the EA2 strategy following application of the MRP was estimated as follows:

1. Number of subjects (OHT and control) improving $=15$ (out of 219). Estimated specificity $=(204 / 219) \times 100=93.2 \%$ ( $95 \%$ confidence interval 88.7 to $95.9 \%$ ).

2. Number of controls progressing $=2$ (out of 21). Estimated specificity $=(19 / 21) \times 100=90.5 \% \quad(95 \%$ confidence interval 68.2 to $98.3 \%)$.

Application of the MRP to the EA2 strategy resulted in an increased number of OHT subjects identified as progressing by HRT compared with the previously published results for the 320 RP, $24.8 \%$ of OHT subjects compared with $16.2 \%$ $\left(\mathrm{p}=0.046\right.$, Fisher exact test). ${ }^{6}$ The agreement between progression identified by EA2 using either the MRP or the $320 \mathrm{RP}$ and

Table 2 Baseline demographics and characteristics of subjects included in the progression analysis

\begin{tabular}{lcc}
\hline & Ocular hypertension & Control \\
\hline No of subjects & 198 & 21 \\
Age (years) & $60(32$ to 79$)$ & 65 (41 to 77$)$ \\
Length of follow-up (years) & $6.0(2.3$ to 7.2$)$ & $5.3(3.1$ to 6.8$)$ \\
No of Heidelberg Retina Tomograph & $10(5$ to 16$)$ & 9 (8 to 11) \\
examinations & $17(5$ to 33$)$ & 9 (7 to 14) \\
No of visual-field examinations & $+0.1(+3.0$ to -2.7$)$ & $+0.1(+2.6$ to -2.4$)$ \\
Baseline mean defect (dB) & $1.24(0.63$ to 2.31$)$ & $1.35(0.86$ to 2.51$)$ \\
Baseline global rim area (mm $\left.{ }^{2}\right)$ & $20(7$ to 186$)$ & 23 (9 to 80$)$ \\
Image quality throughout study (mean & & \\
pixel height SD) & &
\end{tabular}

Values are given as the median (range). progression identified by AGIS criteria is illustrated in a Venn diagram (fig 2). Of the 43 subjects who progressed according to the AGIS criteria, $70 \%$ also progressed by RA using the MRP, compared with $56 \%$ who progressed using the 320RP. This difference was not significant ( $p=0.265$, Fisher exact test).

\section{DISCUSSION}

The MRP was designed with the intention of combining positive attributes of both the SRP and the $320 \mathrm{RP}$, while at the same time compensating for their respective shortcomings. The $320 \mathrm{RP}$ is anchored to a reference ring located in the image periphery. As such, the height of the reference surface (the retina in the image periphery) is likely to be more stable than the reference surface for the SRP. The SRP is anchored at the temporal disc margin, a location which may shift posteriorly as glaucoma progresses and is subject to artefact in the surface height determination near the Elschnig ring. The SRP does, however, have an advantage over the $320 \mathrm{RP}$ in that it can accommodate the large degree of morphological variation of the $\mathrm{ONH}$, such as oblique insertion. The $320 \mathrm{RP}$, by contrast, will be prone to generating inaccurate stereometric parameter measurements when applied to such ONHs.

The results of this study indicate that the $320 \mathrm{RP}$ and MRP generate more repeatable RA measurements than the SRP, regardless of image quality. The differences in RA repeatability between the MRP and the $320 \mathrm{RP}$ are equivocal. Our results

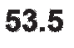

62.1

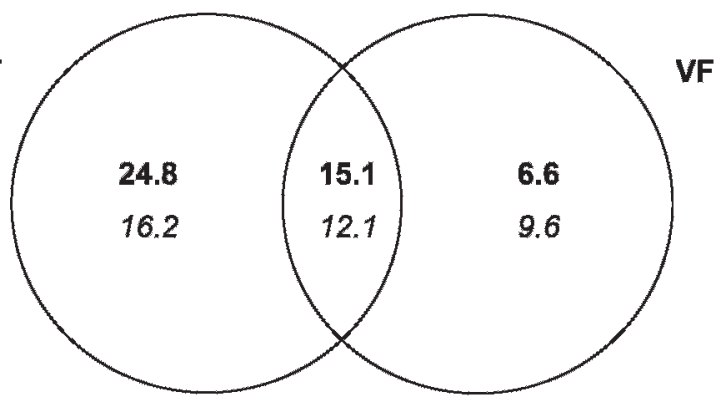

Figure 2 Venn diagram depicting the percentage of ocular hypertensive subjects (of 198) progressing by Heidelberg Retina Tomograph (HRT) Event Analysis 2 (EA2) and by AGIS visual-field (VF) criteria. Values in bold are for the Moorfields Reference Plane, and values in italics are for the 320 reference plane. ${ }^{6}$ The estimated specificity for the HRT criteria is approximately $93 \%$. 
suggest that the SRP has a greater tendency to generate "noisy" RA measurements and should therefore not be considered for use when using RA to monitor progression. This does not, however, preclude the application of the SRP for other purposes; it is important to note that the Moorfields Regression Analysis algorithm uses RA measurements generated using the SRP. ${ }^{2}$

Alternative progression algorithms, which are stereometricparameter-independent have been proposed. One such algorithm is topographical change analysis (TCA), which is incorporated into the current Explorer software. ${ }^{23}$ Our group has also described a method, statistic image mapping (SIM), which has been adapted from neuroimaging techniques. ${ }^{24}$ Both TCA and SIM measure progression by monitoring the pixel-based height change. Unlike RA, the anatomical correlates of SIM and TCA are less easily understood. HRT RA change has a direct anatomical corollorary, which is well understood and may be observed both by clinical examination and by assessment of optic disc stereo-photographs. It is possible that surface height changes within the disc margin, identified by either TCA or SIM, may reflect deformation at the level of the lamina cribrosa, but this has yet to be proven. ${ }^{25}$ TCA has been shown to have poor agreement as regards progression with disc stereo-photographs, ${ }^{26}$ whereas RA-based techniques have a slightly better agreement with disc photography. ${ }^{27}$ It should be stressed that stereo-photography is not an absolute gold standard for glaucomatous progression; therefore, subjects identified as progressing by TCA or SIM but not by photographic examination may very well still be progressing, but in a fashion which cannot be appreciated using the latter technique. As most of our understanding regarding progressive structural changes in glaucoma has been based on careful clinical observations, we would encourage the continued use of stereometric parameterbased (particularly RA) progression algorithms in practice. This recommendation is not exclusive, however, as SIM and TCA are likely to pick up a proportion of genuine progressors which are different from those identified by RA change.

The MRP identified more OHT subjects as progressing than the $320 \mathrm{RP}$, with a similar estimated specificity. The MRP showed improved agreement with VF progression, although this was not statistically significant. The estimated specificity range for EA2 using the $320 \mathrm{RP}(94.1-95.2 \%)$ is slightly higher than that generated for EA2 using the MRP (90.5-93.2\%); given the number of subjects included in these estimates, it is likely that there is no real difference in specificity; however, small changes in specificity can have a large effect on the "positive hit rate." Given the similar values obtained for our two different approaches to obtain proxy measures of specificity, it is reasonable to postulate that the specificity of the criteria applied to the MRP- and the $320 \mathrm{RP}$-generated RA are very similar. The comparison between progression rates using the different RPs is therefore fair, and the observed differences in "positive hit rate" are not simply the result of different specificity values. These results suggest that, at a similar level of specificity, the MRP will identify more subjects as progressing than with the $320 \mathrm{RP}$ and that these subjects are more likely to be genuine progressors. In our previous report we highlighted the higher "signal to noise" ratio of the MRP, and this most probably accounts for the improved detection of progression over the $320 \mathrm{RP}$, rather than RA repeatability which is similar between the two planes. Although these results require confirmation based on further prospective longitudinal studies, the apparent improved performance of the MRP over the $320 \mathrm{RP}$ in detecting RA progression supports its clinical application in the longitudinal detection of structural change using HRT stereometric parameters.
Funding: NGS is funded by a Royal College of Ophthalmologists/Pfizer fellowship and through an unrestricted grant from Heidelberg Engineering. DFG-H has received research support from Heidelberg Engineering, OptoVue and Carl Zeiss Meditec and has acted as a consultant for Carl Zeiss Meditec.

Competing interests: DFG-H has acted as a consultant to Carl Zeiss Meditec. Ethics approval: Ethics approval was provided by Moorfields Eye Hospital Ethics Committee.

Provenance and peer review: Not commissioned; externally peer reviewed.

\section{REFERENCES}

1. Deleon-Ortega JE, Arthur SN, McGwin G Jr, et al. Discrimination between glaucomatous and nonglaucomatous eyes using quantitative imaging devices and subjective optic nerve head assessment. Invest Ophthalmol Vis Sci 2006;47:3374-80.

2. Wollstein G, Garway-Heath DF, Hitchings RA. Identification of early glaucoma cases with the scanning laser ophthalmoscope. Ophthalmology 1998;105:1557-63.

3. Burgansky-Eliash Z, Wollstein G, Bilonick RA, et al. Glaucoma detection with the Heidelberg retina tomograph 3. Ophthalmology 2007;114:466-71.

4. Ferreras A, Pablo LE, Larrosa JM, et al. Discriminating between normal and glaucoma-damaged eyes with the Heidelberg Retina Tomograph 3. Ophthalmology 2008; 115: 775-81.

5. Artes PH, Chauhan BC. Longitudinal changes in the visual field and optic disc in glaucoma. Prog Retin Eye Res 2005;24:333-54

6. Fayers T, Strouthidis NG, Garway-Heath DF. Monitoring glaucomatous progression using a novel Heidelberg Retina Tomograph event analysis. Ophthalmology 2007;114:1973-80.

7. Kamal DS, Garway-Heath DF, Hitchings RA, et al. Use of sequential Heidelberg retina tomograph images to identify changes at the optic disc in ocular hypertensive patients at risk of developing glaucoma. Br J Ophthalmol 2000;84:993-8.

8. Kamal DS, Viswanathan AC, Garway-Heath DF, et al. Detection of optic disc change with the Heidelberg retina tomograph before confirmed visual field change in ocular hypertensives converting to early glaucoma. Br J Ophthalmol 1999;83:290-4.

9. Strouthidis NG, Scott A, Peter NM, et al. Optic disc and visual field progression in ocular hypertensive subjects: detection rates, specificity, and agreement. Invest Ophthalmol Vis Sci 2006:47:2904-10.

10. Tan JC, Hitchings RA. Approach for identifying glaucomatous optic nerve progression by scanning laser tomography. Invest Ophthalmol Vis Sci 2003;44:2621-6.

11. Strouthidis NG, White ET, Owen VM, et al. Factors affecting the test-retest variability of Heidelberg retina tomograph and Heidelberg retina tomograph II measurements. Br J Ophthalmol 2005;89:1427-32.

12. Strouthidis NG, White ET, Owen VM, et al. Improving the repeatability of Heidelberg retina tomograph and Heidelberg retina tomograph II rim area measurements. Br J Ophthalmol 2005;89:1433-7.

13. Tan JC, Garway-Heath DF, Hitchings RA. Variability across the optic nerve head in scanning laser tomography. Br J Ophthalmol 2003;87:557-9.

14. Breusegem C, Fieuws S, Stalmans I, et al. Variability of the standard reference height and its influence on the stereometric parameters of the Heidelberg Retina Tomograph 3. Invest Ophthalmol Vis Sci 2008;49:4881-5.

15. Poli A, Strouthidis NG, Ho T, et al. Analysis of HRT images: comparison of reference planes. Invest Ophthalmol Vis Sci 2008;49:3970-5.

16. British Standards Institution. Precision of test methods 1. Guide for the determination and reproducibility for a standard test method; BS 5497, 1979

17. Kamal D, Garway-Heath D, Ruben S, et al. Results of the betaxolol versus placebo treatment trial in ocular hypertension. Graefes Arch Clin Exp Ophthalmol 2003;241:196-203.

18. AGIS. Advanced Glaucoma Intervention Study. 2. Visual field test scoring and reliability. Ophthalmology 1994;101:1445-55.

19. Mayama C, Araie M, Suzuki Y, et al. Statistical evaluation of the diagnostic accuracy of methods used to determine the progression of visual field defects in glaucoma. Ophthalmology 2004;111:2117-25

20. Zahari M, Mukesh BN, Rait JL, et al. Progression of visual field loss in open angle glaucoma in the Melbourne Visual Impairment Project. Clin Exp Ophthalmol 2006;34:20-6

21. Vesti $\mathbf{E}$, Johnson CA, Chauhan BC. Comparison of different methods for detecting glaucomatous visual field progression. Invest Ophthalmol Vis Sci 2003;44:3873-9.

22. Zeger SL, Liang KY. Longitudinal data analysis for discrete and continuous outcomes. Biometrics 1986;42:121-30.

23. Chauhan BC, Wade BJ, Hamilton DC, et al. Technique for detecting serial topographic changes in optic disc and peripapillary retina using scanning laser tomography. Invest Ophthalmol Vis Sci 2000;41:775-82.

24. Patterson AJ, Garway-Heath DF, Strouthidis NG, et al. A new statistical approach for quantifying change in series of retinal and optic nerve head topography images. Invest Ophthalmol Vis Sci 2005:46:1659-67.

25. Yang H, Downs JC, Girkin C, et al. 3-D histomorphometry of the normal and early glaucomatous monkey optic nerve head: lamina cribrosa and peripapillary scleral position and thickness. Invest Ophthalmol Vis Sci 2007;48:4597-607.

26. Kourkoutas D, Buys YM, Flanagan JG, et al. Comparison of glaucoma progression evaluated with Heidelberg retina tomograph II versus optic nerve head stereophotographs. Can J Ophthalmol 2007;42:82-8.

27. O'Leary N, Mansberger SL, Twa MD, et al. Glaucomatous progression in series of steveo-paired photographs and Heidelberg Retina Tomography images. Invest Ophthalmol Vis Sci 2008;49:E-Abstract 5431. 